\title{
Is diabetes an acquired disorder of reactive glucose metabolites and their intermediates?
}

\author{
T. Fleming • J. Cuny • G. Nawroth $•$ Z. Djuric • \\ P. M. Humpert • M. Zeier • A. Bierhaus • P. P. Nawroth
}

Received: 26 September 2011 / Accepted: 13 December 2011 /Published online: 22 January 2012

(C) Springer-Verlag 2012

\begin{abstract}
Aims/hypothesis We hypothesised that diabetic patients would differ from those without diabetes in regard to the handling of glucose-derived reactive metabolites, evidenced by triosephosphate intermediates $\left(\mathrm{TP}_{\mathrm{INT}}\right)$ and methylglyoxal $(\mathrm{MG})$, irrespective of the type of diabetes, plasma glucose level or $\mathrm{HbA}_{1 \mathrm{c}}$ value.

Methods To test this hypothesis, erythrocytes were isolated from patients with type $1(n=12)$ and type $2(n=12)$ diabetes with varying blood glucose and $\mathrm{HbA}_{1 \mathrm{c}}$ levels. These were then compared with erythrocytes isolated from individuals without diabetes $(n=10)$, with respect to MG, as determined by HPLC, and $\mathrm{TP}_{\mathrm{INT}}$, as determined by endpoint enzymatic assays.

Results The concentrations of intracellular $\mathrm{TP}_{\mathrm{INT}}$ and $\mathrm{MG}$ were significantly elevated in erythrocytes from diabetic patients. Normalisation of either $\mathrm{TP}_{\mathrm{INT}}$ or $\mathrm{MG}$ to intracellular glucose concentration (nmol glucose/mgHb) confirmed that erythrocytes from diabetic patients accumulated more reactive metabolites than did those from healthy controls.

Conclusions/interpretation Diabetic patients can be characterised by an increased formation of $\mathrm{TP}_{\mathrm{INT}}$ and $\mathrm{MG}$. The 25fold increase of MG in type 1 and the 15-fold increase in type 2 diabetes, together with a several-fold increase in $\mathrm{TP}_{\mathrm{INT}}$ and decreased glyceraldehyde-3-phosphate dehydrogenase activity even under normal glucose conditions, imply that normalising glucose level cannot completely prevent late
\end{abstract}

T. Fleming and J. Cuny contributed equally to this study.

T. Fleming $\cdot$ J. Cuny $\cdot$ G. Nawroth $\cdot$ Z. Djuric $\cdot$ P. M. Humpert $\cdot$

M. Zeier $\cdot$ A. Bierhaus $(\bowtie) \cdot$ P. P. Nawroth

Department of Medicine I and Clinical Chemistry,

University of Heidelberg,

INF 410 ,

69120 Heidelberg, Germany

e-mail: angelika.bierhaus@med.uni-heidelberg.de diabetic complications until this acquired error of metabolism has been restored.

Keywords AGEs - Glycation · Metabolism · Oxidative stress · Pathophysiology

$\begin{array}{ll}\text { Abbreviations } \\ \text { DHAP } & \text { Dihydroxyacetonephosphate } \\ \text { FDP } & \text { Fructose-1,6-bisphosphate } \\ \text { GAP } & \text { Glyceraldehyde-3-phosphate } \\ \text { GAPDH } & \text { Glyceraldehyde-3-phosphate dehydrogenase } \\ \text { GLO } & \text { Glyoxalase } \\ \text { MG } & \text { Methylglyoxal } \\ \text { TBARS } & \text { Thiobarbituric acid-reactive substance } \\ \text { TP }_{\text {INT }} & \text { Triosephosphate intermediates }\end{array}$

\section{Introduction}

All therapeutic strategies in the treatment of diabetes mellitus rely on the assumption that the toxic effects of high glucose level and an enhanced metabolic flux are responsible for the generation of toxic metabolites and the development and progression of the associated complications [1]. Lowering blood glucose level is expected to reduce late diabetic complications by reducing the substrate flux through glycolysis, and $\mathrm{HbA}_{1 \mathrm{c}}$ is the accepted marker of glycaemia currently used to guide clinical management [2].

Although the level of $\mathrm{HbA}_{1 \mathrm{c}}$ generally correlates with the risk of complications, some patients with good glycaemic control develop severe complications, whereas others whose glycaemic control may be inadequate remain relatively free of complications for a long time [3]. This suggests the involvement of factors other than hyperglycaemia in the 
development of complications, potentially explaining the issue of 'hyperglycaemic memory'.

An enhanced metabolic flux leads to an increase in reactive carbonyl species through the accumulation of triosephosphate intermediates $\left(\mathrm{TP}_{\mathrm{INT}}\right)$. Methylglyoxal (MG), which belongs to a class of reactive carbonyl species known as the alpha-oxoaldehydes or dicarbonyls, is formed from the spontaneous degradation of $\mathrm{TP}_{\mathrm{INT}}$ and is a highly potent glycating agent [4].

In order to better understand the relationship between substrate load and metabolic consequences, erythrocytes were isolated from patients with type 1 and type 2 diabetes with varying blood glucose and $\mathrm{HbA}_{1 \mathrm{c}}$ levels and compared with erythrocytes isolated from non-diabetic individuals with respect to $\mathrm{MG}$ and $\mathrm{TP}_{\text {INT. }}$. Erythrocytes provide an ideal model system for studying the direct consequences of enhanced metabolic flux, as they lack (1) mitochondria, and as such do not suffer from an enhanced production of reactive oxygen species, and (2) a nucleus, and are therefore unable to respond to metabolic stress.

By providing a standardised continental breakfast, the glucose concentration in erythrocytes from healthy controls was elevated to levels seen in moderate to fairly controlled diabetic patients. We predicted that diabetic patients would differ from non-diabetic patients in regard to the handling of glucose-derived metabolites, evidenced by $\mathrm{TP}_{\mathrm{INT}}$ and $\mathrm{MG}$, irrespective of the type of diabetes or the plasma glucose and $\mathrm{HbA}_{1 \mathrm{c}}$ levels.

\section{Methods}

Participants Blood samples were taken from 24 patients with diabetes (12 with type 1 and 12 with type 2 diabetes) attending the diabetes centre of the University Hospital, Heidelberg, Germany. The controls were 10 non-diabetic patients who had been provided with a standardised continental breakfast prior to sampling. The study protocol was approved by the University of Heidelberg Ethics Committee and all patients gave their informed consent. Full patient characteristics are given in Table 1.

Isolation of human erythrocytes Blood samples were collected by venepuncture into EDTA anticoagulant tubes and processed within $30 \mathrm{~min}$ of sampling. Samples were centrifuged $\left(1,200 \mathrm{rpm}, 15 \mathrm{~min}, 4^{\circ} \mathrm{C}\right)$, plasma and white blood cells were removed, and the pellet comprising the packed erythrocytes was washed three times with four volumes of $0.9 \%$ sodium chloride solution. Subsequently, the erythrocytes were lysed in ice-cold water, the membrane fragments were sedimented $\left(18,000 \mathrm{~g}, 10 \mathrm{~min}, 4^{\circ} \mathrm{C}\right)$ and the resulting haemolysate was either stored at $-80^{\circ} \mathrm{C}$ or analysed immediately. All samples were analysed within 1 month following processing.
Metabolic assays To determine the metabolites of the glyoxalase (GLO) system, haemolysate samples were deproteinated using perchloric acid to a final concentration of $0.5 \mathrm{mmol} / \mathrm{l}$. The precipitated protein was removed by centrifugation. For normalisation of metabolite concentration, haemoglobin concentration was determined, prior to deproteinisation, by the Drabkin assay.

$T P_{I N T}$ The concentration of $\mathrm{TP}_{\mathrm{INT}}$ in deproteinated haemolysate samples was determined, in the presence of sodium arsenate, by endpoint enzymatic assay with glyceraldehyde3-phosphate dehydrogenase (GAPDH), triosephosphate isomerase and aldolase, respectively, by fluorimetric detection of NADH [5]. The total amount of $\mathrm{TP}_{\mathrm{INT}}$, referred to as the triosephosphate pool, was calculated using the following formula $[\mathrm{GAP}]+[\mathrm{DHAP}]+(2 \times[\mathrm{FDP}])$, since glyceraldehyde3-phosphate (GAP), dihydroxyacetonephosphate (DHAP) and fructose-1,6-bisphosphate (FDP) were considered to be in dynamic equilibrium [5].

$M G$ The concentration of MG in the erythrocytes was determined by derivatisation with 1,2-diamino-4,5-dimethoxybenzene and HPLC of the quinoxaline adduct by fluorescence detection, as previously described [6].

Statistics All data are expressed as means \pm SEM $(n \geq 10)$. Unpaired, two-tailed Student's $t$ tests were used to determine significance in all comparisons. Significance of the correlations was calculated at the 5\% level (two-tailed $t$ test) after determining the $t$ value $\left(t=|r| / \sqrt{ }\left(1-r^{2}\right) \times \sqrt{ }(n-2)\right.$, where $r$ is the correlation coefficient and $n$ the number of samples) for each correlated data set. Statistical analysis was performed using SPSS software version 15.0 (SPSS, Chicago, IL, USA). A value of $p>0.05$ was considered to be statistically non-significant.

\section{Results}

In postprandial healthy patients with low values of plasma glucose but much higher levels of intracellular glucose/mg $\mathrm{Hb}$ than diabetic patients (Table 1), no correlation was seen between $\mathrm{TP}_{\mathrm{INT}}$ and intracellular glucose (Fig. 1a, white circles). Furthermore, no correlation was observed between MG and intracellular glucose levels (Fig. 1b, white circles). In contrast, diabetic patients showed significant correlations of both $\mathrm{TP}_{\mathrm{INT}}$ (Fig. 1a, black circles) and MG (Fig. 1b, black circles) with intracellular glucose concentrations. There was no difference between patients with type 1 and type 2 diabetes (data not shown). A significant positive correlation between $\mathrm{TP}_{\mathrm{INT}}$ and $\mathrm{MG}$ was also observed in the diabetic patients, but was not present in the healthy patients (data not shown). 
Table 1 Characteristics of healthy controls and diabetic patients
Data are mean \pm SEM

${ }^{*} p<0.05,{ }^{* * *} p<0.001$,

$\dagger p<0.0001$ vs controls. Unless stated, all other characteristics were NS $(p>0.05)$

\begin{tabular}{|c|c|c|c|}
\hline \multirow[t]{2}{*}{ Variable } & \multirow[t]{2}{*}{ Control } & \multicolumn{2}{|l|}{ Diabetes } \\
\hline & & Type 1 & Type 2 \\
\hline Number & 10 & 12 & 12 \\
\hline Age & $52.8 \pm 16.7$ & $44.4 \pm 17.1$ & $63.1 \pm 9.9$ \\
\hline Sex (female/male) & $7 / 3$ & $5 / 7$ & $7 / 5$ \\
\hline BMI & $27.4 \pm 5.6$ & $26.6 \pm 3.9$ & $31.3 \pm 4.4$ \\
\hline $\mathrm{HbA}_{1 \mathrm{c}}(\%)$ & $5.55 \pm 0.14$ & $8.58 \pm 0.64 \dagger$ & $8.17 \pm 0.70 \% * * *$ \\
\hline $\mathrm{HbA}_{1 \mathrm{c}}(\mathrm{mmol} / \mathrm{mol})$ & $37.2 \pm 1.6$ & $70.2 \pm 7.0 \dagger$ & $65.8 \pm 7.6 * * *$ \\
\hline Duration of diabetes (years) & - & $20.3 \pm 3.3$ & $12.5 \pm 3.3$ \\
\hline \multicolumn{4}{|l|}{ Complications (yes/no) } \\
\hline Neuropathy & - & $3 / 9$ & $6 / 6$ \\
\hline Nephropathy & - & $1 / 11$ & $3 / 9$ \\
\hline Retinopathy & - & $3 / 9$ & $3 / 9$ \\
\hline \multicolumn{4}{|l|}{ Medication } \\
\hline Insulin & - & $12 / 12$ & $12 / 12$ \\
\hline Metformin & - & $1 / 12$ & $4 / 12$ \\
\hline Sulfonylurea & - & - & $1 / 12$ \\
\hline Rosiglitazone & - & - & $1 / 12$ \\
\hline Statins & - & $5 / 12$ & $4 / 12$ \\
\hline Antihypertensives & - & $6 / 12$ & $12 / 12$ \\
\hline Aspirin & - & $3 / 12$ & $9 / 12$ \\
\hline Plasma glucose (mmol/l) & $4.95 \pm 0.11$ & $10.32 \pm 1.09 * * *$ & $9.13 \pm 1.40^{*}$ \\
\hline Plasma MG (nmol/1) & $96.3 \pm 9.5$ & $697.4 \pm 61.3 \dagger$ & $599.3 \pm 53.3 \dagger$ \\
\hline Haemolysate glucose (nmol/mg Hb) & $17.37 \pm 0.30$ & $6.56 \pm 1.2 \dagger$ & $13.55 \pm 1.9$ \\
\hline Haemolysate MG (pmol/mg Hb) & $3.43 \pm 0.38$ & $29.4 \pm 7.59 * * *$ & $38.4 \pm 6.05 \dagger$ \\
\hline $\mathrm{TP}_{\mathrm{INT}}(\mathrm{nmol} / \mathrm{mg} \mathrm{Hb})$ & $0.215 \pm 0.02$ & $0.416 \pm 0.10$ & $0.523 \pm 0.07 \dagger$ \\
\hline DHAP (nmol/mg Hb) & $0.051 \pm 0.004$ & $0.084 \pm 0.013^{* * *}$ & $0.126 \pm 0.018 * * *$ \\
\hline FDP (nmol/mg Hb) & $0.056 \pm 0.003$ & $0.108 \pm 0.025$ & $0.127 \pm 0.016^{* * *}$ \\
\hline GAP (nmol/mg Hb) & $0.053 \pm 0.004$ & $0.100 \pm 0.027$ & $0.144 \pm 0.021 * * *$ \\
\hline \multicolumn{4}{|l|}{ Glucose normalised } \\
\hline $\mathrm{TP}_{\mathrm{INT}}\left(\mathrm{nmol}[\mathrm{nmol} \text { glucose }]^{-1}[\mathrm{mg} \mathrm{Hb}]^{-1}\right)$ & $0.012 \pm 0.003$ & $0.067 \pm 0.03 \dagger$ & $0.045 \pm 0.02 * * *$ \\
\hline $\begin{array}{l}\text { Haemolysate MG } \\
\qquad\left(\mathrm{nmol}[\mathrm{nmol} \text { glucose }]^{-1}[\mathrm{mg} \mathrm{Hb}]^{-1}\right)\end{array}$ & $0.0002 \pm 0.0001$ & $0.005 \pm 0.002 \dagger$ & $0.003 \pm 0.001 \dagger$ \\
\hline
\end{tabular}

Normalisation of either $\mathrm{TP}_{\mathrm{INT}}$ or $\mathrm{MG}$ to intracellular glucose concentration ( $\mathrm{nmol}$ glucose $/ \mathrm{mgHb}$ ) revealed that diabetic patients accumulated more reactive metabolites than healthy controls (Fig. 1c,d). For $\mathrm{TP}_{\mathrm{INT}}$, the increase was 5.5-fold in type 1 and threefold in type 2 diabetes, (Fig. 1c), while the amount of MG per nmol glucose/mg $\mathrm{Hb}$ was increased by 25 -fold in type 1 and 15 -fold in type 2 diabetes (Fig. 1d). Determination of thiobarbituric acidreactive substances (TBARS), allowing for an estimation of oxidative stress in erythrocytes, did not show any significant difference between control and diabetic patients (Fig. 1e). In contrast, GAPDH activity levels were significantly decreased in diabetic patients, regardless of the type of diabetes (Fig. 1f). The GAPDH activity of diabetic erythrocytes did not correlate with any of the measured variables (Fig. 1g-j).

\section{Discussion}

The results of this pilot study demonstrate that diabetic patients can be characterised by a dramatic increase in the formation of intracellular $\mathrm{TP}_{\mathrm{INT}}$ and $\mathrm{MG}$ per mol glucose. Thus, toxic metabolites accumulate even at normal glucose concentrations. A positive correlation of these metabolites with intracellular glucose level was observed only in patients with diabetes, and was not present in healthy controls. When compared with healthy controls, erythrocytes from patients with type 1 diabetes demonstrated a pronounced reduction of intracellular glucose levels by $62 \%$ and an increase of the $\mathrm{TP}_{\mathrm{INT}}$ pool by $193 \%$. Erythrocytes from patients with type 2 diabetes showed a $22 \%$ reduction in intracellular glucose concentration and a highly significant increase of the $\mathrm{TP}_{\mathrm{INT}}$ pool (243\% compared with healthy controls). 
a
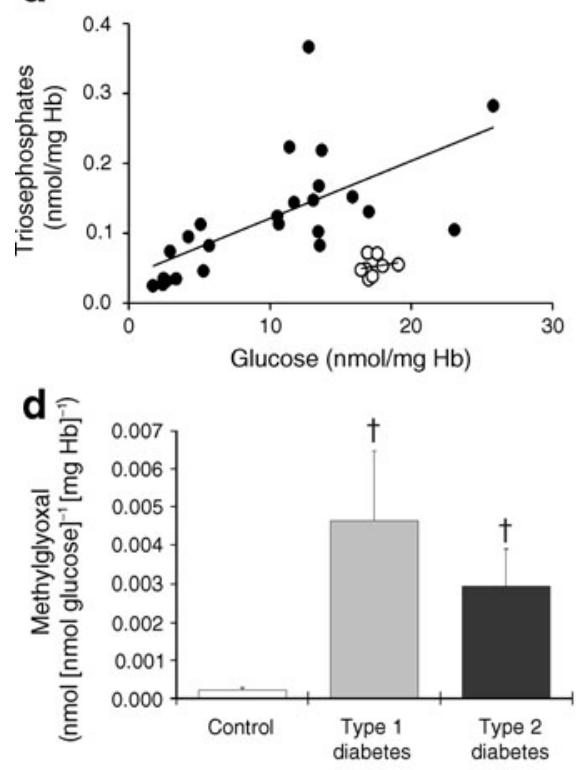

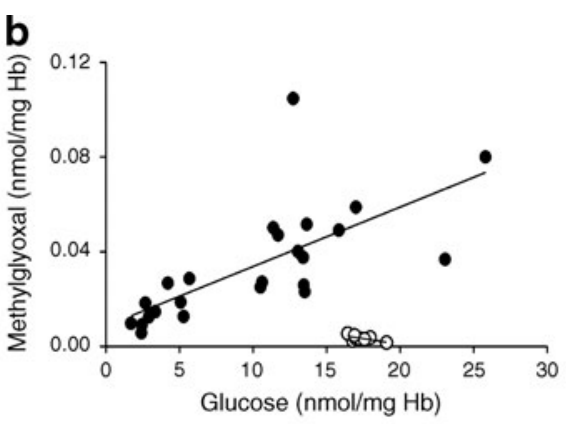

e

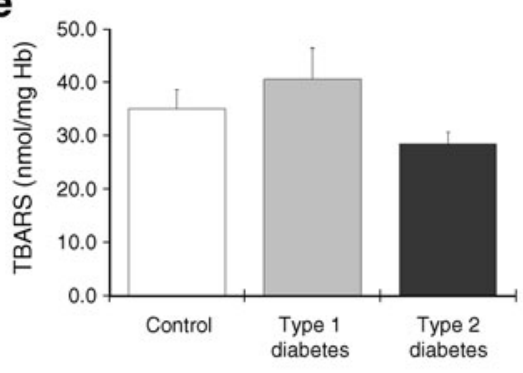

C
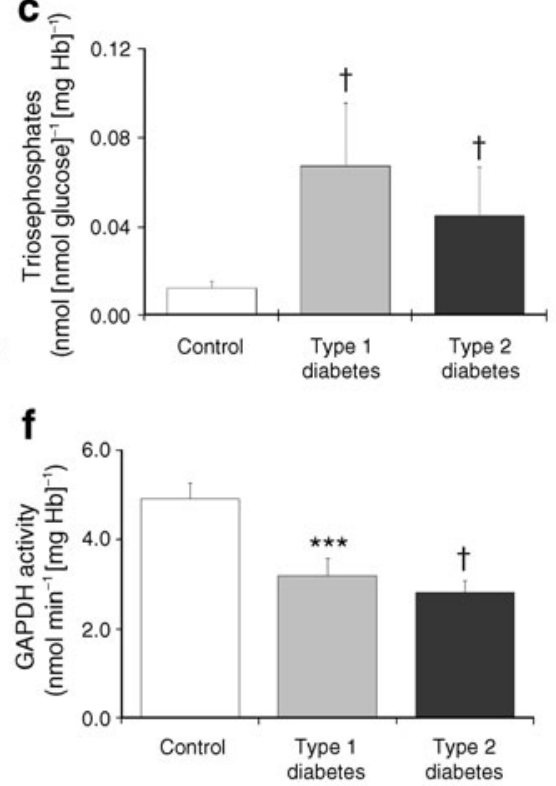

g

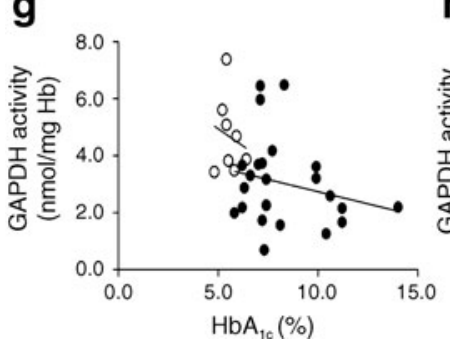

h

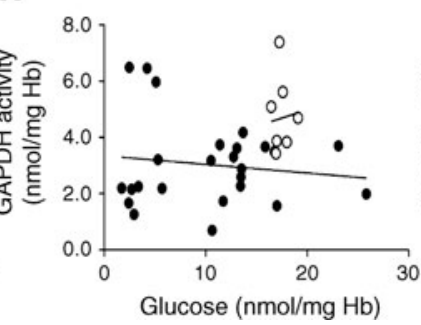

Fig. 1 a Correlation of $\mathrm{TP}_{\mathrm{INT}}$ and glucose in haemolysate from healthy controls $\left(n=10\right.$; white circles; $\left.R^{2}=5 \times 10^{-6}, p=1.0\right)$ and diabetic patients $\left(n=24\right.$, black circles; $\left.R^{2}=0.3669, p=0.002\right)$. b Correlation of MG and glucose in haemolysate from healthy controls $(n=10$, white circles; $\left.R^{2}=0.4567, p=0.07\right)$ and diabetic patients $(n=24$, black circles; $\left.R^{2}=0.4902, p=0.0001\right)$. c $\mathrm{TP}_{\mathrm{INT}}$ normalised to intracellular glucose concentrations in healthy controls $(n=10)$ and patients with type $1(n=$ $12)$ and type $2(n=12)$ diabetes. d MG normalised to intracellular glucose concentrations in healthy controls $(n=10)$ and patients with type $1(n=12)$ and type $2(n=12)$ diabetes. e Concentration of TBARS in haemolysate from healthy controls $(n=10)$ and patients with type 1 $(n=12)$ and type $2(n=12)$ diabetes. f Activity of GAPDH in haemolysate from healthy controls $(n=10)$ and patients with type $1(n=12)$ and type $2(n=12)$ diabetes. g Data represent mean \pm SEM. $* * * p<$ $0.001, \dagger p<0.0001$ vs Controls. Unless stated, all other variables were

It has previously been reported that erythrocytes from diabetic patients show a higher metabolic rate [7] and, as such, the overall glucose turnover in diabetes would be higher. This may explain the significant decrease in intracellular glucose in the erythrocytes of diabetic patients (particularly those with type 1 diabetes) in the presence of a significantly elevated blood glucose level. The observed accumulation in $\mathrm{TP}_{\text {INT }}$ may also reflect an increased glycolytic flux. This is supported by the increased level of FDP observed in all diabetic patients, but was particularly pronounced in patients with type 2 diabetes (Table 1); FDP is enzymatically broken i

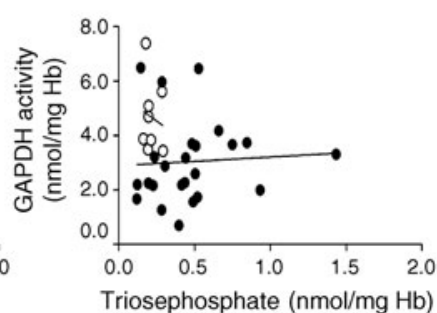

j

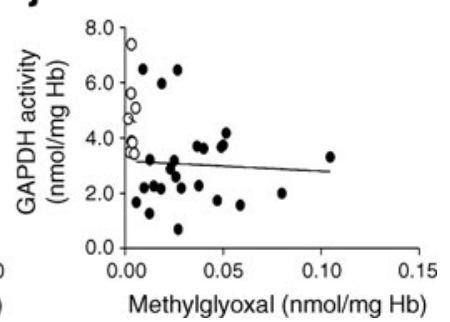

NS $(p>0.05)$. Correlation of GAPDH activity and $\mathrm{HbA}_{1 \mathrm{c}}$ level in haemolysate from healthy controls $\left(n=10\right.$, white circles; $R^{2}=0.0303$; $p=0.680)$ and diabetic patients $\left(n=24\right.$, black circles; $R^{2}=0.0176 ; p=$ 0.285 ). To convert values for $\mathrm{HbA}_{1 \mathrm{c}}$ in $\%$ into $\mathrm{mmol} / \mathrm{mol}$, subtract 2.15 and multiply by 10.929 . h Correlation of GAPDH activity and intracellular glucose level in haemolysate from healthy controls $(n=10$, white circles; $\left.R^{2}=0.0055 ; p=0.861\right)$ and diabetic patients $(n=24$, black circles; $\left.R^{2}=0.0176 ; p=0.537\right)$. i Correlation of GAPDH activity and $\mathrm{TP}_{\mathrm{INT}}$ in haemolysate from healthy controls $\left(n=10\right.$, white circles; $R^{2}=$ $0.0209 ; p=0.733)$ and diabetic patients $\left(n=24\right.$, black circles; $R^{2}=$ 0.0037; $p=0.777)$. $\mathbf{j}$ Correlation of GAPDH activity and MG level in haemolysate from healthy controls $\left(n=10\right.$, white circles; $R^{2}=0.0027$; $p=0.902)$ and diabetic patients $\left(n=24\right.$, black circles; $R^{2}=0.0032$; $p=0.794$ )

down to either GAP or DHAP and therefore reflects the initial metabolic flux through glycolysis.

The accumulation of GAP (Table 1) is indicative of a decreased conversion of GAP to 1,3-bisphosphoglyceric acid by GAPDH. Excessive GAP, converted to DHAP by triosephosphate isomerase, will promote MG formation. This is supported by our findings, in which a 25 -fold increase of MG in erythrocytes in patients with type 1 and a 15 -fold in the erythrocytes of those with type 2 diabetes was observed.

Further analysis of the biochemical components of MG metabolism showed that there were no differences 
in erythrocyte glutathione levels between control and diabetic patients (data not shown). There was also no correlation between MG levels and albuminuria (data not shown), excluding impaired renal function as an underlying cause of the MG accumulation in erythrocytes.

Moreover, and in line with previous studies [8], the activity of GLO-1, the major enzyme involved in detoxifying $M G$, was even higher in erythrocytes from patients with diabetes than in those from healthy controls. In addition, the level of D-lactate, the stable end-product of MG detoxification by the GLO system, was increased only 1.5 -fold in diabetic erythrocytes. Thus, a reduced or defective detoxification by the GLO-1 system is unlikely to account for the increased MG levels observed in erythrocytes from diabetic patients. Other pathways resulting in an increased formation of reactive metabolites, which have yet to be discovered, might at least in part underlie the development of complications in both types of diabetes.

As the level of GAP in type 1 and 2 diabetic patients is elevated (Table 1), the accumulation of $\mathrm{TP}_{\mathrm{INT}}$ might be caused through a reduction or inhibition of GAPDH, an enzyme previously shown to be highly sensitive to inactivation through metabolically derived post-translational modifications [1]. The downregulation of GAPDH by radical oxygen species has been shown to be a key initiator in activating the molecular pathways underlying the development of diabetic complications in mitochondria-bearing nucleated cells [1]. GAPDH can also be inactivated by MG [9] and act as an independent regulator of intracellular MG [10]. However, in the present study, GAPDH activity did not correlate with either TBARS or MG levels, indicating that other mechanisms account for the reduced GAPDH activity observed in erythrocytes from diabetic patients.

In summary, the present study provides strong evidence that patients with diabetes can be characterised by an increased formation of $\mathrm{TP}_{\mathrm{INT}}$ and MG. We hypothesise that those diabetic patients who have an increased accumulation of the $\mathrm{TP}_{\mathrm{INT}}$ and $\mathrm{MG}$ are those patients who are prone to the development of late diabetic complications, despite good glycaemic control. To test this hypothesis, large prospective studies need to be initiated in which both $\mathrm{TP}_{\mathrm{INT}}$ and $\mathrm{MG}$ are measured in parallel with the activity of key erythrocyte enzymes involved in the pentose shunt and in glycolysis. Through a better understanding of these differences, it will be possible to better differentiate those patients who have a higher risk of developing complications. In addition, this will provide novel therapeutic strategies for minimising the production of glucotoxic intermediates.

Funding This work was supported by grants of the Deutsche Forschungsgemeinschaft (BI-1281/3-1 to A. Bierhaus; NA 138/7-1 to P.P. Nawroth), the Dietmar-Hopp-Stiftung (to A. Bierhaus and P.P. Nawroth) and the Network Aging Research (to A. Bierhaus).

Contribution statement TF, JC and GN performed the experiments, analysed the data and were involved in data interpretation and drafting the article. $\mathrm{ZD}, \mathrm{PMH}$ and $\mathrm{MZ}$ contributed to interpretation of data and revised the article critically for important intellectual content. $\mathrm{AB}$ and PPN designed and supervised the project, were responsible for data interpretation and wrote and edited the manuscript. All authors approved the final version of the manuscript to be published.

Duality of interest The authors declare that there is no duality of interest associated with this manuscript.

\section{References}

1. Brownlee M (2001) Biochemistry and molecular cell biology of diabetic complications. Nature 414:813-820

2. Diabetes Control and Complications Trial Research Group (1993) The effect of intensive treatment of diabetes on the development and progression of long-term complications in insulin-dependent diabetes mellitus. N Engl J Med 329:977-986

3. Lachin JM, Genuth S, Nathan DM, Zinman B, Rutledge BN, DCCT/EDIC Research Group (2008) Effect of glycemic exposure on the risk of microvascular complications in the diabetes control and complications trial-revisited. Diabetes 57:995-1001

4. Richard JP (1993) Mechanism for the formation of methylglyoxal from triosephosphates. Biochem Soc Trans 1:549-553

5. Beutler E (1984) Red cell metabolism. A manual of biochemical methods, 3rd edn. Grune \& Stratton, New York, USA

6. McLellan AC, Phillips SA, Thornalley PJ (1992) The assay of methylglyoxal in biological systems by derivatization with 1,2diamino-4,5-dimethoxybenzene. Anal Biochem 206:17-23

7. Tegos C, Bulter E (1980) Red cell glycolytic intermediates in diabetic patients. J Lab Clin Med 96:85-89

8. McLellan AC, Thornalley PJ, Benn J, Sonksen PH (1994) Glyoxalase system in clinical diabetes mellitus and correlation with diabetic complications. Clin Sci (Lond) 87:21-29

9. Lee HJ, Howell SK, Sanford RJ, Beisswenger PJ (2005) Methylglyoxal can modify GAPDH activity and structure. Ann N Y Acad Sci 1043:135-145

10. Beisswenger PJ, Howell SK, Smith K, Szewegold BS (2003) Glyceraldehyde-3-phosphate dehydrogenase activity as an independent modifier of methylglyoxal levels in diabetes. Biochim Biophys Acta 1637:98-106 